\title{
High-Intensity Focused Ultrasound or Uterine Artery Embolization combined with ultrasound-guided dilatation and curettage in Cesarean scar pregnancy: a retrospective cohort study
}

\section{yanqiong Gan}

Affiliated Hospital of North Sichuan Medical College https://orcid.org/0000-0002-8549-5510

\section{Yuqin Zhou}

Affiliated Hospital of North Sichuan Medical College

\section{Yanlin Wang}

Affiliated Hospital of North Sichuan Medical College

Qiao Jing

Affiliated Hospital of North Sichuan Medical College

\section{Feng Zhang}

Affiliated Hospital of North Sichuan Medical College

Qi Shi ( $\nabla$ shiqistone@163.com )

Affiliated Hospital of North Sichuan Medical College

\section{Research Article}

Keywords: cesarean scar pregnancy, high intensity focused ultrasound, uterine artery embolization, ultrasound-guided dilatation and curettage

Posted Date: March 2nd, 2022

DOI: https://doi.org/10.21203/rs.3.rs-802864/v2

License: (c) (1) This work is licensed under a Creative Commons Attribution 4.0 International License. Read Full License 


\section{Abstract}

\section{Background}

Cesarean scar pregnancy (CSP) is a rare and new form of ectopic pregnancy. With increasing rate of cesarean delivery worldwide during the decades, the incidence of CSP increases as well. It may cause massive hemorrhage, uterine rupture, placenta percreta, hysterectomy or even maternal death. This study is to compare the efficacy of high-intensity focused ultrasound (HIFU) and uterine artery embolization (UAE) associated with ultrasound-guided dilatation and curettage (UGDC) in three types of CSP.

\section{Methods}

403 CSP patients were treated with UGDC after pretreatment. Among them, 288 patients chose HIFU before UGDC, while the others $(n=115)$ chose UAE. The body mass index (BMI), gravidity, parity, the number of cesarean delivery, the interval of previous cesarean delivery (PCD), the length, thickness and width of uterus and gestational sac, fetal cardiac activity, the types of CSP, the baseline of beta-human chorionic gonadotropin ( $\beta-H C G)$, the value of $\beta-H C G$ before and 24 hours after pretreatment, hospital expenditure, the value of hemoglobin before and 24 hours after dilatation and curettage (D\&C) and severe complications were collected and compared between the two groups.

\section{Results}

All patients were successfully treated without severe complications. There was no significant difference in maternal age, BMI, gravidity, parity, the number of cesarean delivery, the interval of PCD, the volume of uterus and gestational sac, fetal heart activity, types of CSP and baseline serum $\beta$-HCG level between the two groups. The median hospital expenditure and blood loss were less in HIFU group and the median decline rate of $\beta$-HCG was higher in HIFU group ( $p=0.000, p=0.000, p=0.006$, respectively). 39 (13.54\%) patients in HIFU group, while 21 (18.26\%) cases in UAE group used Foley balloon to control the vaginal bleeding.

\section{Conclusion}

Both HIFU and UAE combined with UGDC have high successful rate in the treatment of CSP. While, HIFU followed by UGDC might be better for less hospital expenditure, blood loss and higher decline rate of $\beta$ HCG.

\section{Background}

Cesarean scar pregnancy (CSP) is a rare and new form of ectopic pregnancy. It was defined as the gestational sac embedded into the scar of previous cesarean delivery (PCD)[1]. With increasing rate of cesarean delivery worldwide during the decades[2-4], the incidence of CSP increases as well. Larsen et al.[5] reported the first case of cesarean scar ectopic pregnancy in 1978. Since then, only 19 cases had been reported in English medical literature until 2001. However, in the last 20 years, a great number of 
articles about CSP have been submitted. Currently, the estimated incidence of CSP ranges from 1:2216 to $1: 1800$ of all conceives and $6.1 \%$ of all ectopic pregnancies in women with at least one cesarean delivery[6, 7]. However, the true incidence is still unknown cause misdiagnosed. Because of the abundant heterogeneous blood vessels and the weak myometrium in the scar area, CSP may cause severe complications, such as massive hemorrhage, uterine rupture, placenta percreta, hysterectomy and even maternal death[8-11]. In a recent review[12], the authors summarized 14 treatment modalities. However, the optimal management is still unclear. High intensity focused ultrasound (HIFU) or uterine artery embolization (UAE) associated with ultrasound-guided dilatation and curettage (UGDC) are generally accepted. However, most researches were small samples or just included only one type of CSP and the results existed differences[13-15]. And there is still no enough evidence to confirm which therapy is the best. The aim of our retrospective cohort study is to compare the efficacy of pretreatment - HIFU or UAE combined with UGDC in three types of CSP. Our study demonstrated that HIFU associated with UGDC was more effective and economical than UAE.

\section{Materials And Methods}

\section{Ethics approval}

The study received the approval from the Ethics Committee of Affiliated Hospital of North Sichuan Medical College.

\section{Participants}

From January 2015 to December 2020, 502 patients with CSP were managed in our department. Finally, 403 patients were included in our study. The flow chart was shown in Fig. 1.

Inclusion criteria: (1) patients had a history of cesarean delivery, (2) had amenorrhea and the blood pregnancy test was positive, (3) gestational age less than 12 weeks, (4) the ultra-sonographic findings met the diagnostic criteria[16], (5) with intact medical record.

Exclusion criteria: (1) patients associated with uterine fibroids or other gynecological disorders, (2) with acute vaginitis or pelvic inflammatory diseases, (3) with other severe disease such as failure of heart, liver or kidney, (4) with unstable hemodynamics, (5) the histopathology after operation indicated without trophoblastic tissue, (6) had been treated in other hospital.

The information of each patient, including age, body mass index (BMI), gravidity, parity, the number of cesarean delivery (CD), the interval of previous cesarean delivery (PCD), the length (D1), thickness (D2) and width (D3) of uterus and gestational sac, fetal cardiac activity, the types of CSP, the baseline of betahuman chorionic gonadotropin ( $\beta$-HCG), the value of $\beta$-HCG before and 24 hours after pretreatment, hospital expenditure, the value of hemoglobin before and 24 hours after dilatation and curettage (D\&C) and severe complications, was collected from the medical records.

The uterine size and gestational sac volume were calculated with the following formula: 
$V=(1 / 6) \times \boldsymbol{\Pi} \times \mathrm{D} 1 \times \mathrm{D} 2 \times \mathrm{D} 3$. The decline ration of beta-human chorionic gonadotropin $(\beta-H C G)$ was calculated with the following formula: (the $\beta$-HCG level before pretreatment - the $\beta$-HCG level 24 hours after pretreatment) / the $\beta$-HCG level before pretreatment. The blood loss was showed by the decline of hemoglobin and calculated with the following formula: the value of hemoglobin before $D \& C$ - the value of hemoglobin 24 hours after D\&C. The severe complications were defined as blood loss $\geq 30 \mathrm{~g} / \mathrm{L}$ and/or laparoscopy and/or laparotomy and/or hysterectomy and/or blood transfusion.

The classification criteria for CSP[17, 18]: Type I: 1 . only a small part of the gestational sac morphed into the niche of cesarean section; 2 . the myometrium thickness between the gestational sac and the posterior wall of the bladder was $>3 \mathrm{~mm} ; 3$. without or minimal blood flow was detected around the gestational sac (Fig. 2A). Type II: 1. part of the gestational sac morphed into the niche of cesarean section; 2 . the myometrium thickness between the gestational sac and the posterior wall of the bladder was $\leq 3 \mathrm{~mm} ; 3$. moderate blood flow was detected around the gestational sac (Fig. 2B). Type III: 1. most of the gestational sac implanted into the niche; 2 . the myometrium thickness between the gestational sac and the posterior wall of the bladder was $<2 \mathrm{~mm} ; 3$. marked blood flow was detected around the gestational sac (Fig. 2C).

\section{High intensity focused ultrasound}

Enhanced magnetic resonance imaging (MRI) was used to confirm the size, location and blood supply of gestational sac. Bowel preparation was performed using polyethylene glycol-electrolyte powder. Each patient was shaved the lower abdominal area. A catheter was inserted into bladder to control bladder size and push the bowel away from the acoustic pathway.

The procedure was performed by two experienced gynecologists using the JC200 focused ultrasound tumour therapeutic system (Chongqing HIFU Technology Co., Ltd., Chongqing, China). This system is integrated with real-time monitoring ultrasound (MyLab 70, Esaote, Genova, Italy). Focused ultrasound energy was produced by a $20-\mathrm{cm}$ diameter transducer with frequency of $1.0 \mathrm{MHz}$, which was located in a reservoir filled with cold degassed and degreased water[19]. Patients were conscious sedation and prone on the HIFU system, with the abdominal wall immerged in the sealed reservoir. During treatment, the focus was placed close to the embedding area of the gestational sac in the center slice at the beginning of the HIFU ablation procedure. Cessation of HIFU ablation occurred when: (1) obvious grey scale change in the target area was observed; (2) blood flow signal of gestational sac embedding area disappeared or was significantly reduced in the colour Doppler. SonoVue was injected $5 \mathrm{~min}$ pre-and immediately posttreatment to evaluate the ablation effect. The therapeutic effect was defined as: (1) a non-perfused volume was observed in gestational sac embedding area; (2) blood supply around the gestational sac, especially the embedding area, disappeared or significantly reduced[20].

\section{Uterine artery embolization}

Antibiotic was used with a single dose via venous before UAE. The operation of UAE was performed by two experienced interventional radiologists using digital subtraction arteriography (DSA) (AXIOM-Artis-FA; 
Siemens AG, Munich, Germany). Patients were supine on the UAE system. Local anesthesia was performed at the right groin area. After anesthesia, catheter was punctured via the right femoral artery using the Seldinger technique. DSA was used to confirm the uterine artery blood flow, the location of the gestational sac and its vessels, then embolized bilateral uterine arteries with absorbable gelatin compressed sponges (1-1.4mm, Ailikang, Hangzhou, China). The operation was ceased after another angiography indicated uterine artery blocked and staining disappeared[21, 22].

\section{Ultrasound-guided suction curettage}

An experienced gynecologist performed the procedure $24 \mathrm{~h}$ after pretreatment. Antibiotic was used $30 \mathrm{~min}$ before the surgery. $10 \mathrm{U}$ oxytocin via $500 \mathrm{ml}$ intravenous injection drip was routinely used during the operation. The operation was performed under general anesthesia with lithotomy position and suprapubic ultrasonography guidance. A vacuum aspirator (Yuyue, Jiangsu, China) was used to aspirate the whole gestational sac avoiding perforate the previous cesarean scar. The tissues were sent for pathological examination (Video). If intractable uterine hemorrhage happened, an 18-Fr gauge Foley catheter balloon tamponade with $100 \mathrm{ml}$ saline was used for $12 \mathrm{~h}$ (Fig. 3). Additional surgery was performed for uncontrolled bleeding.

\section{Statistical analysis}

SPSS 24.0 was used for statistical analysis. Normal distribution data were represented by means $\pm S D$ and analyzed with $T$ test. Abnormal distribution data were represented by median and inter-quartile range (P25-P75) and analyzed with the Mann-Whitney $U$ test. Qualitative data were analyzed with Chi-square test. A two-side $P<0.05$ was considered statistically significant difference.

\section{Results}

\section{Demographic characteristics}

There was no significant difference in maternal age, BMI, gravidity, parity, the number of CD, the interval of PCD, the volume of uterus and gestational sac, fetal heart activity, types of CSP and baseline serum $\beta$ HCG level.

\section{Clinical outcomes}

All patients who received UGDC after pretreatment were cured successfully. The median hospital expenditure and blood loss were less in HIFU group and the median decline rate of $\beta$-HCG was higher in HIFU group ( $p=0.000, p=0.000, p=0.006$, respectively). 39 (13.54\%) patients in HIFU group, while 21 (18.26\%) in UAE group used Foley balloon to control the vaginal bleeding. No patient need more surgeries or blood transfusion in the two groups. The results were showed in Table 1. 
Table 1

The baseline characteristics and outcomes between HIFU group and UAE group.

\begin{tabular}{|c|c|c|c|c|}
\hline characteristics and outcomes & HIFU group $(n=288)$ & UAE group $(n=115)$ & $\begin{array}{l}\mathrm{T} / \mathrm{Z} / \mathrm{X}^{2} \\
\text { value }\end{array}$ & $\begin{array}{l}P \\
\text { value }\end{array}$ \\
\hline Age, years (mean $\pm S D)$ & $32.32 \pm 4.89$ & $32.39 \pm 5.12$ & -0.138 & 0.890 \\
\hline BMI, (mean \pm SD) & $22.51 \pm 3.47$ & $22.17 \pm 3.28$ & 0.890 & 0.374 \\
\hline Gravidity, p50(p25, p75) & $4.5(3,6)$ & $5(3,6)$ & -0.662 & 0.508 \\
\hline Parity, p50(p25, p75) & $1(1,2)$ & $2(1,2)$ & -0.905 & 0.365 \\
\hline CD number, p50(p25, p75) & $1(1,2)$ & $1(1,2)$ & -1.222 & 0.222 \\
\hline PCD interval, years (mean \pm SD) & $4.5(3,8)$ & $4(3,7)$ & -0.854 & 0.393 \\
\hline $\begin{array}{l}\text { The volume of uterus, } \mathrm{cm}^{3} \\
\text { p50(p25, p75) }\end{array}$ & $\begin{array}{l}142.37(106.60 \\
185.27)\end{array}$ & $\begin{array}{l}\text { 139.13(113.76, } \\
169.60)\end{array}$ & -0.977 & 0.328 \\
\hline $\begin{array}{l}\text { The volume of gestational sac, } \\
\mathrm{cm}^{3} \mathrm{p} 50(\mathrm{p} 25, \mathrm{p} 75)\end{array}$ & $2.51(0.90,7.70)$ & $3.19(1.06,7.48)$ & -0.015 & 0.988 \\
\hline Fetal heart exist detected, $n(\%)$ & $111(38.54)$ & 43(37.39) & 0.046 & 0.830 \\
\hline Types of CSP, $n(\%)$ & & & 0.914 & 0.633 \\
\hline Type I & $106(36.80)$ & $48(41.74)$ & & \\
\hline Type II & 165(57.29) & $60(52.17)$ & & \\
\hline Type III & $17(5.90)$ & $7(6.09)$ & & \\
\hline $\begin{array}{l}\text { Baseline of HCG, IU/L p50(p25, } \\
\text { p75) }\end{array}$ & $\begin{array}{l}34782.50(13973.97 \\
70628.50)\end{array}$ & $\begin{array}{l}41880.00(13179.00 \\
71467.00)\end{array}$ & -0.119 & 0.905 \\
\hline $\begin{array}{l}\text { Hospital expenditure, yuan } \\
\text { p50(p25, p75) }\end{array}$ & $\begin{array}{l}14747.03(13860.13 \\
16828.82)\end{array}$ & $\begin{array}{l}\text { 18365.94(17314.77, } \\
\text { 19300.29) }\end{array}$ & -7.258 & 0.000 \\
\hline Blood loss, g/L (mean \pm SD) & $13(6,18)$ & $18(9,25)$ & -4.257 & 0.000 \\
\hline $\begin{array}{l}\text { The decline of HCG, } p 50(p 25 \text {, } \\
\text { p75) }\end{array}$ & $0.81(0.65,0.92)$ & $0.72(0.59,0.86)$ & -2.756 & 0.006 \\
\hline Foley balloon implanted, $n(\%)$ & $39(13.54)$ & $21(18.26)$ & 1.444 & 0.229 \\
\hline
\end{tabular}

HIFU high intensity focused ultrasound; UAE uterine artery embolization; $S D$ standard deviation; $B M I$ body mass index; $C D$ cesarean delivery; $P C D$ previous cesarean delivery; $C S P$ cesarean scar pregnancy

\section{Discussion}

Along with the increased cesarean section rate and improved ultrasonic techniques, the detection rate of CSP is consistent rising. The estimated incidence is around 1.7 per 1000 pregnancies[10]. The natural 
ending of CSP is unpredictable, for it has a high risk of developing into miscarriage, preterm birth, placenta accreta spectrum, uterine rupture and even life-threatening hemorrhage or hysterectomy[23]. Early termination was recommended in CSP. HIFU or UAE combined with UGDC are two main treatments for CSP. Currently, there is still no enough evidence to confirm which therapy is the best. Our study indicated that both HIFU and UAE associated with UGDC have high successful rate, while HIFU combined with UGDC was more effective and economical.

HIFU technique can lead to coagulative necrosis with a heat over $65^{\circ} \mathrm{C}[24]$ and damage small vessels[25], while UAE technique can embolize bilateral uterine arteries and block the blood flow of uterus and embryo. These could be the theories that the decreased blood loss in the therapy of CSP.

Our study expanded and supported the previous studies. In the latest meta-analysis[26], 388 CSP patients were treated by HIFU and UGDC, while 327 CSP patients were treated by UAE and UGDC. The results indicated that HIFU group had less blood loss, adverse effects and shorter hospital stay. Our research enhanced the evidence. Liu CN et al.[13] performed HIFU combined with hysteroscopy-guided suction curettage in 103 CSP patients and their successful rate was $98.06 \%$. But they just included type I CSP, while we included all types. In another study[15], the successful rate of HIFU combined with UGDC was only $70.37 \%$, and the authors indicated that HIFU was good for CSP patients with a gestational age of < 55 days and a maximum gestational sac diameter of $<30 \mathrm{~mm}$. While, all patients in different diameters got cured in our research. And we also use volume to demonstrate the size of gestational sac. To our limited knowledge, we thought that the volume of gestational sac could better reflect the real gestational age and the difficulty of the operation.

Different with the traditional classification of CSP[27], we classified CSP into three types according to the site of implantation, the myometrium thickness between the gestational sac and the posterior wall of the bladder and the blood flow of gestational sac. The thinner of the myometrium, the more dangerous for uterine rupture. And the more blood flow means the higher risk for intraoperative bleeding. It can better reflect the risk and difficulty of therapy. Our study showed that HIFU group had less hospital expenditure, blood loss and higher decline rate of $\beta$-HCG. Patient who chose HIFU need not hospitalize, so the hospital expenditure was lower. And the thermal energy killed the embryo and ablated the blood vessels directly and the blood vessels shut and collapsed 24 hours after HIFU therapy, as the blood loss was less and the decline rate of $\beta$-HCG. Our study also has limitations. We lack of long-term follow-ups and reproductive outcomes in the current study, while relevant data is being collected and analyzed.

\section{Conclusions}

The present study finds that both high-intensity focused ultrasound and uterine artery embolization combined with ultrasound-guided dilatation and curettage have high successful rate in the treatment of cesarean scar pregnancy. While, HIFU might be better for less hospital expenditure, blood loss and higher decline rate of $\beta$-HCG. 


\section{Abbreviations}

CSP: cesarean scar pregnancy; $P C D$. previous cesarean delivery; HIFU: high intensity focused ultrasound; UAE: uterine artery embolization; UGDC. ultrasound-guided dilatation and curettage; $B M$ : body mass index; $C D$. cesarean delivery; $\beta-H C G$ : beta-human chorionic gonadotropin; $D \& C$. dilatation and curettage; $M R l$ : magnetic resonance imaging; $D S A$ : digital subtraction arteriography.

\section{Declarations}

\section{Availability of data and materials}

The datasets used and /or analyzed during the current study are available from the corresponding author on reasonable request.

\section{Ethics approval and consent to participate}

The study received the approval from the Ethics Committee of Affiliated Hospital of North Sichuan Medical College. The informed consent is not applicable but we phoned every patients and got agreements. The verbal consent was approved by the ethics committee .

\section{Consent for publication}

Not applicable.

\section{Competing interests}

None declared.

\section{Funding}

No funding source was involved.

\section{Author contribution}

YG: Data analysis and manuscript writing. YZ and YW: Data collection. QJ and FZ: images and vedio processing. QS: Project development. All authors have read, revised and approved the manuscript.

\section{Acknowledgements}

We would like to thank the academic support of Science and Technology of North Sichuan Medical College and each participants.

\section{References}


1. Ash A, Smith A, Maxwell D. Caesarean scar pregnancy. BJOG: an international journal of obstetrics gynaecology. 2007;114(3):253-63.

2. Diejomaoh MFE, Al-Jassar W, Bello Z, Karunakaran K, Mohammed A. The Relevance of the Second Cesarean Delivery in the Reduction of Institutional Cesarean Delivery Rates. Medical principles and practice: international journal of the Kuwait University. Health Science Centre. 2018;27(6):555-61.

3. Morton R, Burton AE, Kumar P, et al. Cesarean delivery: Trend in indications over three decades within a major city hospital network. Acta obstetricia et gynecologica Scandinavica. 2020;99(7):909-16.

4. Li HT, Luo S, Trasande L, et al. Geographic Variations and Temporal Trends in Cesarean Delivery Rates in China, 2008-2014. Jama. 2017;317(1):69-76.

5. Larsen JV, Solomon MH. Pregnancy in a uterine scar sacculus-an unusual cause of postabortal haemorrhage. A case report. South African medical journal = Suid-Afrikaanse tydskrif vir geneeskunde. 1978;53(4):142-143.

6. Seow KM, Huang LW, Lin YH, Lin MY, Tsai YL, Hwang JL. Cesarean scar pregnancy: issues in management. Ultrasound in obstetrics gynecology: the official journal of the International Society of Ultrasound in Obstetrics Gynecology. 2004;23(3):247-53.

7. Jurkovic D, Hillaby K, Woelfer B, Lawrence A, Salim R, Elson CJ. First-trimester diagnosis and management of pregnancies implanted into the lower uterine segment Cesarean section scar. Ultrasound in obstetrics gynecology: the official journal of the International Society of Ultrasound in Obstetrics Gynecology. 2003;21(3):220-7.

8. O'Neill SM, Khashan AS, Kenny LC, et al. Caesarean section and subsequent ectopic pregnancy: a systematic review and meta-analysis. BJOG: an international journal of obstetrics gynaecology. 2013;120(6):671-80.

9. Silver RM. Implications of the first cesarean: perinatal and future reproductive health and subsequent cesareans, placentation issues, uterine rupture risk, morbidity, and mortality. Seminars in perinatology. 2012;36(5):315-23.

10. Rotas MA, Haberman S, Levgur M. Cesarean scar ectopic pregnancies: etiology, diagnosis, and management. Obstetrics gynecology. 2006;107(6):1373-81.

11. Maymon R, Halperin R, Mendlovic S, et al. Ectopic pregnancies in Caesarean section scars: the 8 year experience of one medical centre. Human reproduction (Oxford England). 2004;19(2):278-84.

12. Birch Petersen K, Hoffmann E, Rifbjerg Larsen C, Svarre Nielsen H. Cesarean scar pregnancy: a systematic review of treatment studies. Fertility sterility. 2016;105(4):958-67.

13. Liu CN, Tang L, Sun Y, Liu YH, Yu HJ. Clinical outcome of high-intensity focused ultrasound as the preoperative management of cesarean scar pregnancy. Taiwan J Obstet Gynecol. 2020;59(3):38791.

14. Huo X, Xie Y, Yang L, Deng K, Liu Y. Doppler Ultrasound Evaluation of the Efficacy of High Intensity Focused Ultrasound in Treatment of Cesarean Section Scar Pregnancy. Journal of the College of Physicians Surgeons-Pakistan: JCPSP. 2020;30(3):240-4. 
15. Fang S, Zhang P, Zhu Y, Wang F, He L. A Retrospective Analysis of the Treatment of Cesarean Scar Pregnancy by High-Intensity Focused Ultrasound, Uterine Artery Embolization and Surgery. Frontiers in surgery. 2020;7:23.

16. Timor-Tritsch IE, Monteagudo A, Santos R, Tsymbal T, Pineda G, Arslan AA. The diagnosis, treatment, and follow-up of cesarean scar pregnancy. American journal of obstetrics gynecology. 2012;207(1):44.e41-13.

17. Li J, Weilin C, Zhou Yingfang. The consensus of management of cesaresan section pregnancy (2016). Chinese journal of obstetrics and gynecology. 2016;51(08):568-572.

18. Adler DD, Carson PL, Rubin JM, Quinn-Reid D. Doppler ultrasound color flow imaging in the study of breast cancer: preliminary findings. Ultrasound Med Biol. 1990;16(6):553-9.

19. Chen $Y$, Jiang J, Zeng Y, et al. Effects of a microbubble ultrasound contrast agent on high-intensity focused ultrasound for uterine fibroids: a randomised controlled trial. International journal of hyperthermia: the official journal of European Society for Hyperthermic Oncology North American Hyperthermia Group. 2018;34(8):1311-5.

20. Zhang Y, Zhang C, He J, Bai J, Zhang L. The impact of gestational sac size on the effectiveness and safety of high intensity focused ultrasound combined with ultrasound-guided suction curettage treatment for caesarean scar pregnancy. International journal of hyperthermia: the official journal of European Society for Hyperthermic Oncology North American Hyperthermia Group. 2018;35(1):2917.

21. Li Y, Lu L, Wang W, Sun J, Zhang X, Huang X. Retrospective study of patients with cesarean scar pregnancies treated by uterine artery chemoembolization and curettage. Int J Gynaecol Obstet. 2018;143(2):172-7.

22. Zhang S, Yan H, Ji WT. Uterine artery embolization combined with intra-arterial MTX infusion: its application in treatment of cervical pregnancy. Archives of gynecology obstetrics. 2016;293(5):1043-7.

23. Morlando M, Buca D, Timor-Tritsch I, et al. Reproductive outcome after cesarean scar pregnancy: A systematic review and meta-analysis. Acta obstetricia et gynecologica Scandinavica. 2020;99(10):1278-89.

24. Zhang L, Chen WZ, Liu YJ, et al. Feasibility of magnetic resonance imaging-guided high intensity focused ultrasound therapy for ablating uterine fibroids in patients with bowel lies anterior to uterus. Eur J Radiol. 2010;73(2):396-403.

25. Wu F, Wang ZB, Zhu H, et al. Feasibility of US-guided high-intensity focused ultrasound treatment in patients with advanced pancreatic cancer: initial experience. Radiology. 2005;236(3):1034-40.

26. Xiao X, Feng Z, Li T, Yi B, Zhang S, Wang W. Comparing the Efficacy and Safety of High-Intensity Focused Ultrasound and Uterine Artery Embolization in Caesarean Scar Pregnancy: A Meta-analysis. Advances in therapy. 2019;36(6):1314-25.

27. Vial Y, Petignat P, Hohlfeld P. Pregnancy in a cesarean scar. Ultrasound in obstetrics \& gynecology: the. official journal of the International Society of Ultrasound in Obstetrics Gynecology. 
2000;16(6):592-3.

\section{Figures}

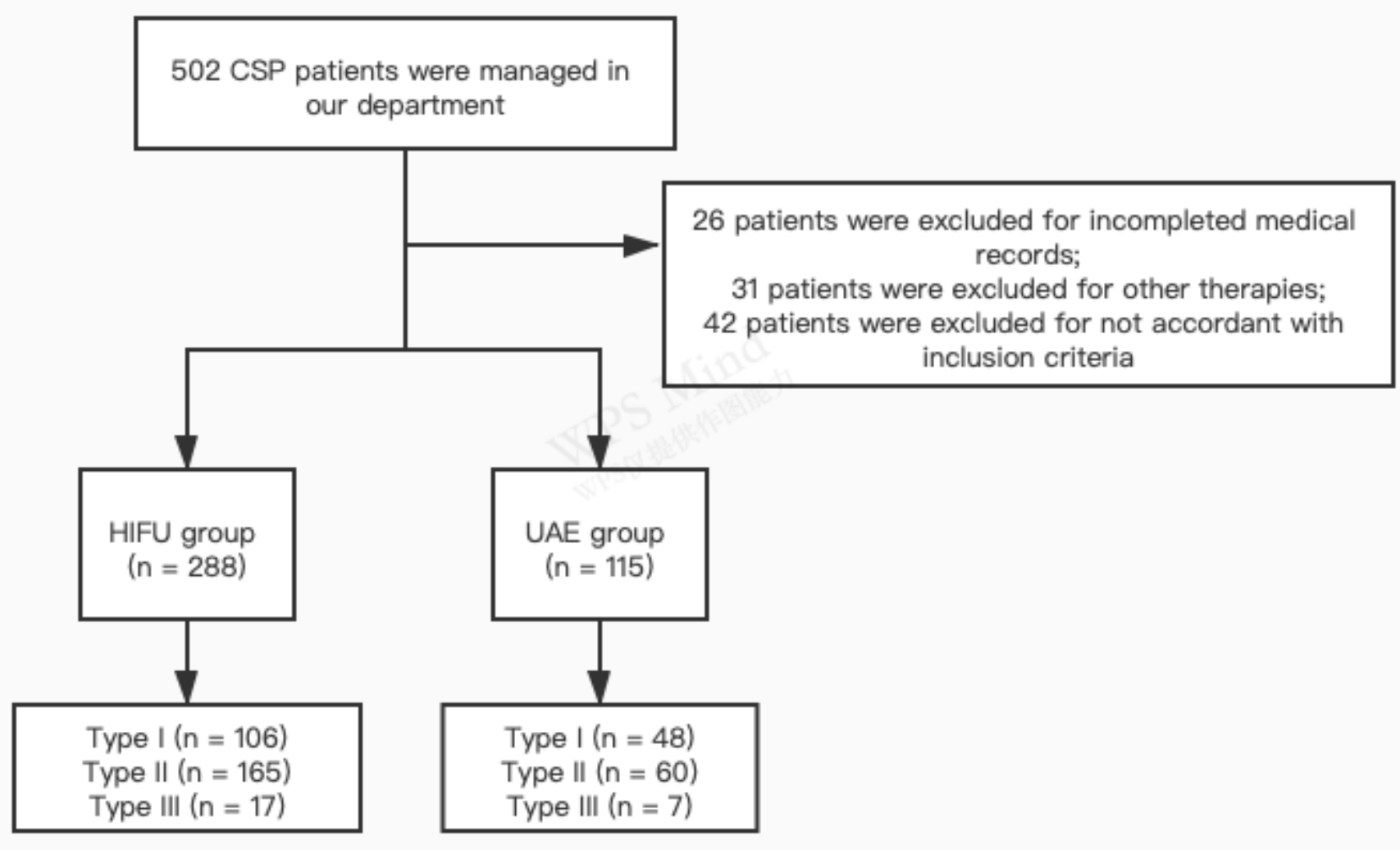

Figure 1

The flow chart of patient screening.

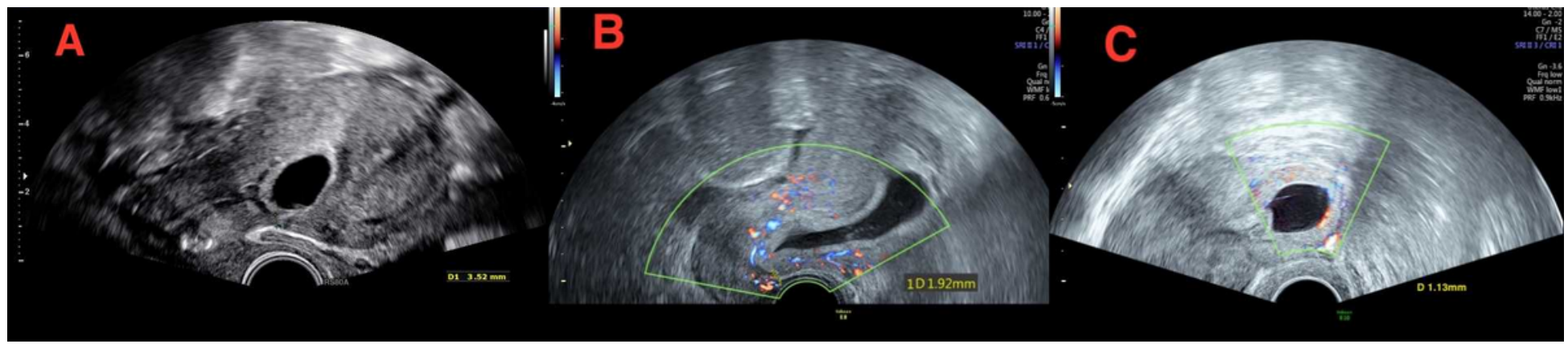

Figure 2

A (1). only a small part of the gestational sac morphed into the niche of cesarean section; (2). the myometrium thickness between the gestational sac and the posterior wall of the bladder was $3.52 \mathrm{~mm}$; (3). no obvious blood flow was detected around the gestational sac. B (1). part of the gestational sac morphed into the niche of cesarean section; (2). the myometrium thickness between the gestational sac 
and the posterior wall of the bladder was $1.92 \mathrm{~mm}$; (3). moderate blood flow was detected around the gestational sac. C (1). most of the gestational sac implanted into the niche; (2). the myometrium thickness between the gestational sac and the posterior wall of the bladder was $1.13 \mathrm{~mm}$; (3). marked blood flow was detected around the gestational sac.

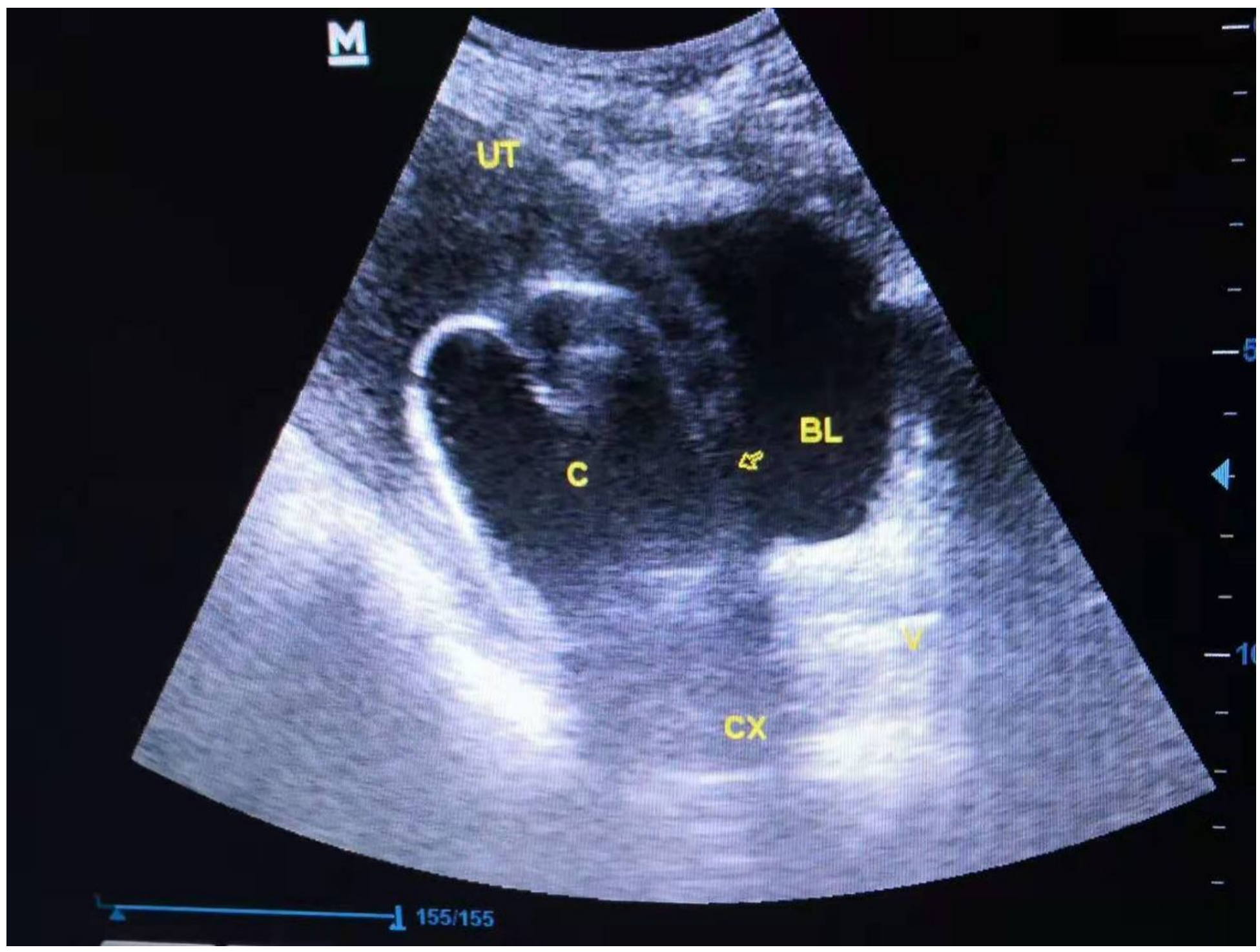

\section{Figure 3}

Foley catheter to control the bleeding.

V vagina; CX cervix; $C$ foley catheter; $B L$ bladder; UT uterus 